\section{(1) \\ CrossMark}

\title{
A scoring system to predict the elevation of mean pulmonary arterial pressure in idiopathic pulmonary fibrosis
}

\author{
Taiki Furukawa ${ }^{1,2}$, Yasuhiro Kondoh ${ }^{1}$, Hiroyuki Taniguchi ${ }^{1}$, Mitsuaki Yagi ${ }^{3}$, \\ Toshiaki Matsuda', Tomoki Kimura', Kensuke Kataoka', Takeshi Johkoh", \\ Masahiko Ando ${ }^{5}$, Naozumi Hashimoto ${ }^{2}$, Koji Sakamoto ${ }^{2}$ and \\ Yoshinori Hasegawa ${ }^{2}$
}

Affiliations: ${ }^{1}$ Dept of Respiratory Medicine and Allergy, Tosei General Hospital, Seto, Japan. ${ }^{2}$ Dept of Respiratory Medicine, Nagoya University Graduate School of Medicine, Nagoya, Japan. ${ }^{3}$ Dept of Respiratory Medicine, National Hospital Organization Higashi Nagoya National Hospital, Nagoya, Japan. ${ }^{4}$ Dept of Radiology, Kinki Central Hospital of Mutual Aid Association of Public Health Teachers, Hyougo, Japan. ${ }^{5}$ Center for Advanced Medicine and Clinical Research, Nagoya University Hospital, Nagoya, Japan.

Correspondence: T. Furukawa, Dept of Respiratory Medicine, Nagoya University Graduate School of Medicine, 65 Tsurumai-cho, Showa-ku, Nagoya, Aichi 466-8550, Japan. E-mail: yxjry228aybb.ne.jp

@ERSpublications

A simple scoring system comprising three variables can predict the likelihood of elevation of MPAP in IPF http://ow.ly/kZjA30haKvo

Cite this article as: Furukawa T, Kondoh $\mathrm{Y}$, Taniguchi H, et al. A scoring system to predict the elevation of mean pulmonary arterial pressure in idiopathic pulmonary fibrosis. Eur Respir J 2018; 51: 1701311 [https:// doi.org/10.1183/13993003.01311-2017].

ABSTRACT Elevated mean pulmonary arterial pressure (MPAP; $\geqslant 21 \mathrm{mmHg}$ ) is sometimes seen in patients with idiopathic pulmonary fibrosis (IPF) and has an adverse impact upon survival. Although early diagnosis is crucial, there is no established screening tool that uses a combination of noninvasive examinations.

We retrospectively analysed IPF patients at initial evaluation from April 2007 to July 2015 and, using logistic regression analysis, created a screening tool to identify elevated MPAP. Internal validation was also assessed for external validity using a bootstrap method.

Using right-heart catheterisation (RHC), elevation of MPAP was determined to be present in 55 out of 273 patients. Multivariate models demonstrated that \% predicted diffusing capacity of the lung for carbon monoxide (DLCO) $<50 \%$, ratio of pulmonary artery diameter to aorta diameter (PA/Ao) on computed tomography $(\mathrm{CT}) \geqslant 0.9$ and arterial oxygen tension $\left(\mathrm{PaO}_{2}\right)<80$ Torr were independent predictors. When we assigned a single point to each variable, the prevalence of elevation of MPAP with a score of zero, one, two or three points was $6.7 \%, 16.0 \%, 29.1 \%$ and $65.4 \%$, respectively. The area under curve (AUC) for the receiver operating characteristic (ROC) curve was good at 0.757 (95\% CI 0.682-0.833).

A simple clinical scoring system consisting of \% predicted DLCO, PA/Ao ratio on CT and $\mathrm{PaO}_{2}$ can easily predict elevation of MPAP in patients with IPF.

This article has supplementary material available from erj.ersjournals.com

Received: July 012017 | Accepted after revision: Oct 132017

Support statement: This study was supported by a grant to the Diffuse Lung Disease Research Group from the Japanese Ministry of Health and by AMED under grant number JP17ek0109269. Funding information for this article has been deposited with the Crossref Funder Registry.

Conflict of interest: Disclosures can be found alongside this article at erj.ersjournals.com

Copyright OERS 2018 


\section{Introduction}

Idiopathic pulmonary fibrosis (IPF) is a chronic, progressive fibrotic lung disease of unknown aetiology [1]. Following diagnosis, the median survival time is approximately $2-3$ years and treatment options are limited [1]. Pulmonary hypertension $(\mathrm{PH})$, defined as an elevation of resting mean pulmonary arterial pressure (MPAP) to $\geqslant 25 \mathrm{mmHg}$, is a well-recognised complication in advanced IPF which is associated with higher mortality rates [2-5]. While the upper level of normal for MPAP is $20 \mathrm{mmHg}$, the significance of MPAP levels between 21 and $24 \mathrm{mmHg}$ remains unclear in terms of their prognostic and therapeutic implications [6, 7]. However, KIMURA et al. [8] have demonstrated that, in patients with IPF, even elevation of MPAP $(\geqslant 21 \mathrm{mmHg})$ at initial evaluation indicated a higher mortality rate. TERAMACHI et al. [9] have also revealed that MPAP gradually progresses (the annual change in their patients was $1.8 \mathrm{mmHg}$ per year) and that at initial evaluation MPAP was a significant predictor of mortality. Thus, early detection of elevated MPAP $(\geqslant 21 \mathrm{mmHg})$ is important.

However, the equivocal clinical findings on elevated MPAP in IPF pose a challenge, as symptoms (such as dyspnoea on exertion) are common in both PH and IPF [7]. Thoracic echocardiography is a noninvasive screening tool for PH but it does not show favourable accuracy in IPF [10, 11]. Alternatively, the ratio of pulmonary artery diameter (PA) to ascending aorta diameter (Ao) on computed tomography (CT) of the chest is a useful indicator of $\mathrm{PH}$ [11] and is reportedly a predictor of higher mortality [12]. Other noninvasive predictors of $\mathrm{PH}$ have been reported; however, they have limited discriminative ability for $\mathrm{PH}$ in IPF $[10,13-16]$. Thus, there is a need for reliable criteria to determine when right heart catheterisation (RHC) should be performed in suitable patients.

With this in mind, AlkukHun et al. [11] have attempted to create a noninvasive screening tool for $\mathrm{PH}$ based on the PA/Ao ratio from chest CT in 235 patients with IPF who underwent RHC as part of a lung transplant evaluation. Zisman et al. [17] have also proposed a noninvasive screening tool for $\mathrm{PH}$ using standard lung function measurements; however, these tools are complicated and difficult to use in clinical practice and the study populations include only advanced patients. Therefore, the aim of the present study is to predict elevated MPAP $(\geqslant 21 \mathrm{mmHg})$ in patients with IPF at initial evaluation using a combination of noninvasive examinations.

\section{Material and methods \\ Study subjects}

We retrospectively analysed medical records from 302 consecutive patients with IPF who underwent initial systemic evaluation between April 2007 and July 2015 at Tosei General Hospital (Aichi, Japan). The diagnosis of IPF was made according to the 2011 international consensus guidelines. For patients who initially presented before 2010, the diagnoses were confirmed in May 2015 [1]. Of these 302 patients the following were excluded: 18 patients who declined RHC; four patients who had already been prescribed medication for IPF or PH (i.e. antifibrotic drugs, corticosteroids, immunosuppressants, or drugs for the treatment of $\mathrm{PH}$ ), or had already undergone long-term oxygen therapy, who might thus exhibit treatment bias; and seven patients who had a pulmonary arterial wedge pressure (PAWP) of $>15 \mathrm{mmHg}$ following RHC, indicative of left-heart disease. Consequently, we examined 273 patients with IPF in a derivation cohort and this study was approved by the Tosei General Hospital institutional review board (IRB number 584). The requirement for informed consent was waived other than the initial informed consent for physical examination as part of regular clinical practice.

\section{Data collection}

Clinical data were collected retrospectively by medical chart review. Eligible patients underwent physical examination and assessment of physiological function, exercise capacity (by 6-min walk test (6MWT) and desaturation just after the 6MWT), and RHC at the initial evaluation, as usual for assessment for interstitial pneumonia.

All patients completed pulmonary function tests by spirometry (CHESTAC-55V; Chest, Tokyo, Japan) according to the American Thoracic Society (ATS)/European Respiratory Society (ERS) criteria [18]. Diffusing capacity of the lung for carbon monoxide (DLCO) was also measured (CHESTAC-55V) [19]. The 6MWT was performed according to the ATS/ERS criteria [20] while the RHC procedure was performed according to the most recent international guidelines [6]. A high-resolution computed tomography (HRCT) chest scan, performed within the 3-month window prior to the RHC procedure, was reviewed in a blinded, independent fashion by two of the authors (T. Furukawa and M. Yagi). The diameter of the pulmonary artery (PA) on HRCT was measured at its bifurcation, the largest diameter of the ascending aorta (Ao) was measured at the corresponding level and the PA/Ao ratio was calculated from these measurements. The intra-class correlation coefficient was used to determine the level of agreement between the two readers for the continuous variables of PA, Ao and the PA/Ao ratio. The extent of 
fibrosis and of the low attenuation area (LAA) were scored by a thoracic radiologist with 28 years of experience. Combined pulmonary fibrosis and emphysema (CPFE) was defined as having $\geqslant 10 \%$ LAA in the upper zone on the chest HRCT (supplementary material).

\section{Statistical analysis}

All analyses were performed using IBM SPSS Statistics v. 24 (IBM Corp., Armonk, NY, USA) and R statistical software (The R Project for Statistical Computing, www.r-project.org/). Continuous data were presented as mean $\pm \mathrm{SD}$ or median (range) as appropriate, while categorical variables were reported as frequency (\%). Between-group differences were assessed using a two-sided t-test, Mann-Whitney U-test or Chi-squared test as appropriate, while the results of the regression analyses were presented in terms of the estimated odds ratios with corresponding $95 \%$ confidence intervals.

Predictor variables that were statistically significant were included in a multiple regression model to determine those that could appropriately predict the elevation of MPAP. The use of predictors as continuous variables showed similar statistical significance both before and after categorisation (supplementary table S1). We believe that categorisation of predictor variables can facilitate the application of the score in daily clinical practice. To determine the threshold of a categorical variable to be included in the score, we divided each continuous variable into a binary variable considering the prior literature, the ROC curve analysis for predicting the elevation of MPAP, and the ease-of-use in daily practice. Finally, we selected a threshold for each predictor variable (data not shown) as follows: $\mathrm{PaO}_{2}>80$ Torr [8], forced vital capacity $(\mathrm{FVC})>75 \%$ predicted, DLCO $>50 \%$ predicted $[16,21]$, 6 -min walk distance $(6 \mathrm{MWD})>540 \mathrm{~m}$ [14], lowest arterial oxygen saturation measured by pulse oximetry $\left(\mathrm{SpO}_{2}\right)$ during the $6 \mathrm{MWD}>80 \%$, $\mathrm{PA} /$ Ao ratio $\geqslant 0.9[12]$, and estimated right-ventricular systolic pressure (RVSP) using echocardiography $>30 \mathrm{mmHg}[14,22]$. LAA $\geqslant 10 \%$ in the upper zone was taken as being indicative of the presence of CPFE. Multivariable logistic regression analysis was performed using forward model selection to create a model that best predicted MPAP $\geqslant 21 \mathrm{mmHg}$. To avoid multicollinearity, one part of the highly correlated variables (Pearson's correlation coefficient $>0.7$ ) was excluded in the multivariate analysis. The ROC curve was determined to estimate the AUC of the simplified score. The bootstrap method was used to internally validate this score by sampling with replacement for 1000 iterations ( $p$-values $<0.05$ were considered statistically significant).

\section{Results}

A total of 273 patients were enrolled in the study and the overall prevalence of elevated MPAP and PH confirmed by RHC was $20.1 \%(n=55)$ and $6.6 \%(n=18)$, respectively (figure 1$)$. Patient demographics and other clinical data are given in table 1 . In comparison to the lower MPAP group (MPAP $<21 \mathrm{mmHg}$ ), the higher group (MPAP $\geqslant 21 \mathrm{mmHg}$ ) was characterised by lower $\mathrm{PaO}_{2}, \mathrm{FVC}$ (\% predicted), forced expiratory volume in $1 \mathrm{~s}$ (FEV1; \% predicted), DLCO (\% predicted) and $6 \mathrm{MWD}$, as well as the lowest $\mathrm{SpO}_{2}$ during the $6 \mathrm{MWT}$. Moreover, the higher MPAP group showed a greater extent of LAA, greater presence of CPFE, and greater PA, PA/Ao ratio and estimated RVSP. As there were high degrees of correlation between FVC (\% predicted) and FEV1 (\% predicted) (Pearson correlation coefficient $0.82, \mathrm{p}<0.001$ ) and between PA and the PA/Ao ratio (Pearson correlation coefficient $0.72, \mathrm{p}<0.001$ ), we selected FVC (\% predicted) and the $\mathrm{PA} / \mathrm{Ao}$ ratio as candidate variables for multivariate analysis. In the CT findings, the inter-rater reliability of

FIGURE 1 Distribution of resting mean pulmonary arterial pressure (MPAP) in the study population that underwent right heart catheterisation. Dark vertical bars indicate elevation of MPAP ( $\geqslant 21 \mathrm{mmHg}$ ).

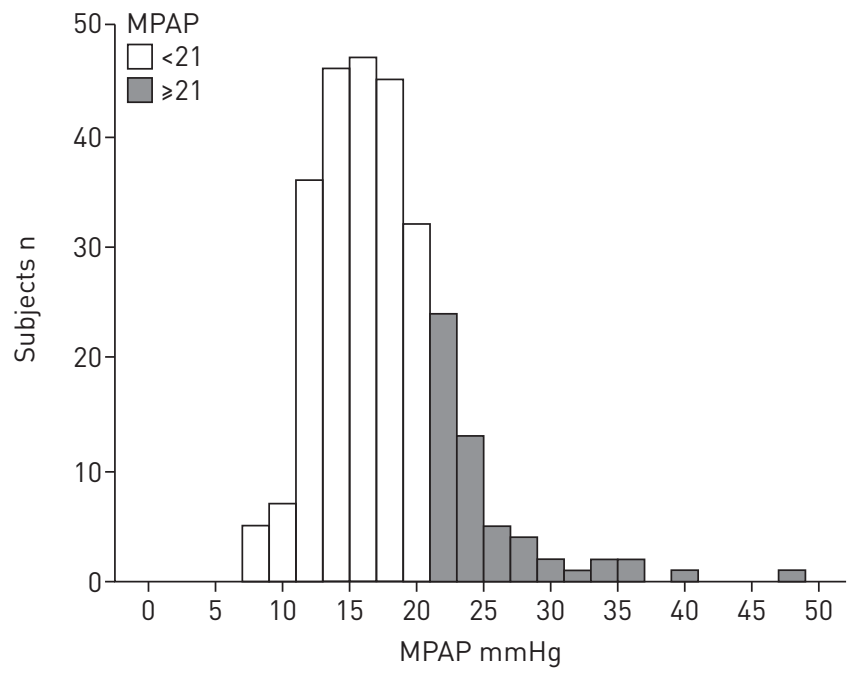


TABLE 1 Baseline characteristics of the study population

\begin{tabular}{|c|c|c|c|}
\hline Characteristic & MPAP $\geqslant 21 \mathrm{mmHg}$ & MPAP $<21 \mathrm{mmHg}$ & p-value \\
\hline Subjects ${ }^{\#}$ & 55 & 218 & \\
\hline Age years & $64.5 \pm 6.8$ & $66.7 \pm 7.8$ & 0.06 \\
\hline Male & $44(80.0)$ & 183 (83.9) & 0.49 \\
\hline BMI $\mathrm{kg} \cdot \mathrm{m}^{-2}$ & $24.7 \pm 4.0$ & $23.6 \pm 3.2$ & 0.05 \\
\hline pack-years & $43.1 \pm 37.4$ & $34.7 \pm 33.5$ & 0.10 \\
\hline mMRC dyspnoea scale & & & $<0.001$ \\
\hline Level 0 & 7 & 72 & \\
\hline Level 1 & 21 & 96 & \\
\hline Level 2 & 14 & 37 & \\
\hline Level 3 & 10 & 11 & \\
\hline Level 4 & 3 & 2 & \\
\hline $\mathrm{PaO}_{2} \mathrm{mmHg}$ & $74.5 \pm 12.1$ & $83.5 \pm 11.3$ & $<0.001$ \\
\hline $\mathrm{BNP} \mathrm{pg} \cdot \mathrm{mL}^{-1}$ & $20.7(10.8-34.9)$ & $17.2(9.7-30.9)$ & 0.23 \\
\hline FVC $\%$ predicted & $75.4 \pm 20.8$ & $82 \pm 19.2$ & 0.03 \\
\hline FEV1 \% predicted & $85.4 \pm 20.0$ & $94.3 \pm 19.9$ & 0.003 \\
\hline FEV1/FVC & $85.7 \pm 8.8$ & $85.1 \pm 7.8$ & 0.60 \\
\hline DLco $\%$ predicted & $46.9 \pm 18.3$ & $64.3 \pm 20.9$ & $<0.001$ \\
\hline 6MWD m & $508 \pm 124$ & $585 \pm 135$ & $<0.001$ \\
\hline Lowest $\mathrm{SpO}_{2} \%$ & $77.5 \pm 10.5$ & $84.3 \pm 8.2$ & $<0.001$ \\
\hline \multicolumn{4}{|l|}{ HRCT findings } \\
\hline PA mm & $28.7 \pm 3.6$ & $26.6 \pm 3.8$ & $<0.001$ \\
\hline Ao mm & $32.6 \pm 3.6$ & $33.4 \pm 3.6$ & 0.11 \\
\hline PA/Ao & $0.89 \pm 0.12$ & $0.80 \pm 0.11$ & $<0.001$ \\
\hline Fibrosis \% & $35.0(24.2-46.7)$ & $31.7(22.5-41.7)$ & 0.11 \\
\hline LAA \% & $1.7(0.0-11.7)$ & $0.0(0.0-3.3)$ & 0.02 \\
\hline$\geqslant 10 \%$ in upper zone & $22(40.0)$ & $54(24.8)$ & 0.02 \\
\hline$\geqslant 25 \%$ in upper zone & $8(14.5)$ & $12(5.5)$ & 0.03 \\
\hline \multicolumn{4}{|l|}{ Echocardiograph findings } \\
\hline Ejection fraction \% & $0.65 \pm 0.12$ & $0.68 \pm 0.06$ & 0.14 \\
\hline $\mathrm{E} / \mathrm{A}$ & $0.47 \pm 0.43$ & $0.65 \pm 0.98$ & 0.18 \\
\hline Estimated RVSP mmHg & $29.6 \pm 20.6$ & $22.8 \pm 16.4$ & 0.01 \\
\hline \multicolumn{4}{|l|}{ RHC findings } \\
\hline MPAP mmHg & $24.9 \pm 5.3$ & $15.0 \pm 2.9$ & $<0.001$ \\
\hline PVR Wood units & $3.0 \pm 1.8$ & $1.7 \pm 0.7$ & $<0.001$ \\
\hline Cardiac index $\mathrm{L} \cdot \mathrm{min}^{-1} \cdot \mathrm{m}^{-2}$ & $3.0 \pm 0.6$ & $3.0 \pm 0.5$ & 0.96 \\
\hline PAWP $\mathrm{mmHg}$ & $10.7 \pm 3.4$ & $6.6 \pm 3.0$ & $<0.001$ \\
\hline
\end{tabular}

Data are presented as $\mathrm{n}, \mathrm{n}(\%)$, mean \pm SD or median (interquartile range), unless otherwise indicated. ${ }^{\#}$ : the total number of subjects (N) was 273. MPAP: mean pulmonary arterial pressure; BMI: body mass index; mMRC: modified Medical Research Council; $\mathrm{PaO}_{2}$ : arterial oxygen tension; BNP: brain natriuretic peptide; FVC: forced vital capacity; FEV1: forced expiratory volume in $1 \mathrm{~s}$; DLCo: diffusing capacity of the lung for carbon monoxide; 6MWD: 6-min walk distance; $\mathrm{SpO}_{2}$ : arterial oxygen saturation measured by pulse oximetry; HRCT: high-resolution computed tomography; PA: pulmonary artery diameter; Ao: ascending aorta diameter; LAA: low attenuation area; E: peak velocity flow in early diastole (E-wave); A: peak velocity flow in late diastole (A-wave); RVSP: right-ventricular systolic pressure; RHC: right heart catheterisation; PVR: pulmonary vascular resistance; PAWP: pulmonary arterial wedge pressure.

TABLE 2 Multivariate logistic regression analysis for elevation of mean pulmonary arterial pressure (MPAP)

\begin{tabular}{lccc} 
Variables $^{*}$ & OR $(95 \% \mathrm{CI})$ & p-value & Points \\
\hline Duco $(\%$ predicted) $<50 \%$ & $3.624(1.763-7.449)$ & $<0.001$ & 1 \\
$\mathrm{PaO}_{2}<80$ Torr & $2.653(1.242-5.665)$ & 0.012 & 1 \\
PA/Ao $>\mathbf{0 . 9}$ & $2.245(1.083-4.656)$ & 0.030 & 1 \\
\hline
\end{tabular}

\#: C-index 0.772 (95\% Cl 0.699-0.845; $\mathrm{p}<0.001)$. D LCo: diffusing capacity of the lung for carbon monoxide; $\mathrm{PaO}_{2}$ : arterial oxygen tension; PA: pulmonary artery diameter; Ao: ascending aorta diameter. 
$\mathrm{PA}, \mathrm{Ao}$ and the PA/Ao ratio was almost perfect, with discrimination of greater than 0.9 in the PA/Ao ratio being substantial (supplementary table S2).

\section{Development of the risk tool}

Based on multivariable analysis (table 2), the candidate variables found to be significantly associated with the elevation of MPAP were DLCO (\% predicted) $\leqslant 50 \%$ (versus $>50 \%)$, PA/Ao ratio $\geqslant 0.9($ versus $<0.9)$ and $\mathrm{PaO}_{2}<80$ Torr (versus $\geqslant 80$ Torr) (C-index $0.772,95 \%$ CI $0.699-0.845 ; \mathrm{p}<0.001$ ). Based on the $\beta$-regression coefficients of the covariates in the final multivariable models, we assigned a score of one point to each covariate depending on the proximity of the coefficient to the nearest whole number, as this would facilitate easy interpretation by clinicians. The individual covariate scores were totalled to obtain an individual risk score ranging from zero to three.

\section{Accuracy and validation of the risk tool}

We created a cumulative graph (figure 2) and plotted the risk scores using the ROC curve (figure 3). In the simplified scores, a score of three points indicated an elevation of MPAP by about $65 \%$ and was associated with an approximately 26-fold probability of elevated MPAP (table 3). A score of zero had a specificity of $54.2 \%$ and a sensitivity of $13.2 \%$, with a positive-predictive value (PPV) of $6.7 \%$ for the elevation of MPAP. By contrast, a score of three had a specificity of $95.8 \%$ and a sensitivity of $32.1 \%$, with a PPV of $65.4 \%$ for the elevation of MPAP (table 4). The AUC of the model was good $(0.757,95 \% \mathrm{CI}$ 0.682-0.833) and the Hosmer-Lemeshow goodness-of-fit test for the derivation cohort was nonsignificant ( $\mathrm{p}=0.58$ ), indicating that the model had a good fit. After internal validation by the bootstrap method, the AUC of the simplified score was 0.756 (95\% CI 0.687-0.825).

\section{Discussion}

We developed a simple, noninvasive screening tool based on $\mathrm{PaO}_{2}$, DLCO (\% predicted) and the PA/Ao ratio derived from HRCT. This tool might be useful in clinical settings because it allows simple, noninvasive selection of patients with elevated MPAP, in whom RHC should be performed, especially those achieving three points in the present scoring system.

A few screening tools have already been developed and Zisman et al. [17, 23] have developed and validated equation 1, which considers room air resting pulse oximetry and the FVC/DLCO ratio, and demonstrated its relatively high negative predictive value (NPV) for PH in advanced IPF patients.

$$
\mathrm{MPAP}=-11.9+0.272 \times S_{\mathrm{pO}_{2}}+0.0659 \times\left(100-S_{\mathrm{pO}_{2}}\right)^{2}+3.06 \times\left(\% \mathrm{FVC} / \% D_{\mathrm{LCO}}\right)
$$

Furthermore, Alkukhun et al. [11] have attempted to create a noninvasive screening tool for PH in IPF as part of the evaluation for lung transplantation; however, they have concluded that noninvasive tests, alone or in combination, are not accurate enough for PH screening in IPF patients. In comparison with these

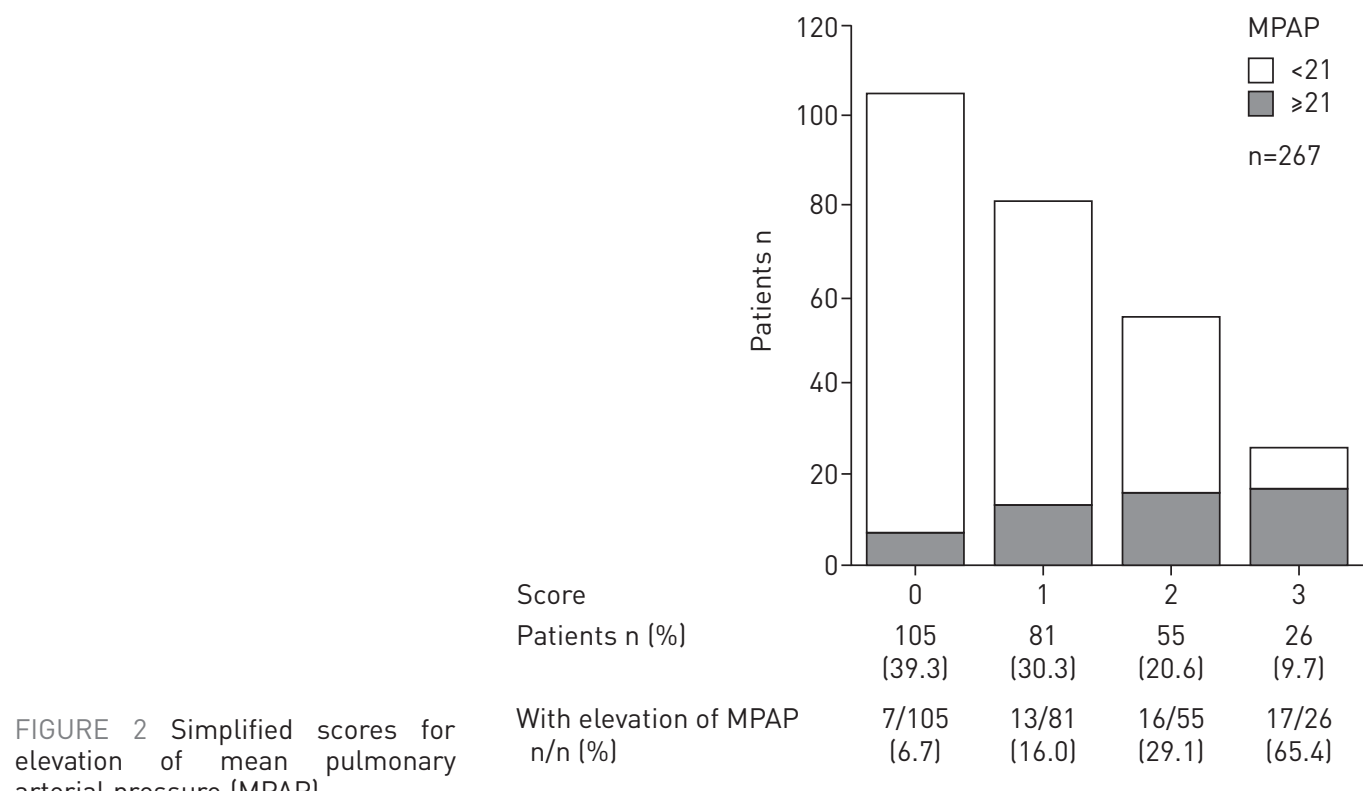


FIGURE 3 Receiver operating characteristic (ROC) curve for the simplified score predicting for elevation of mean pulmonary arterial pressure (MPAP).

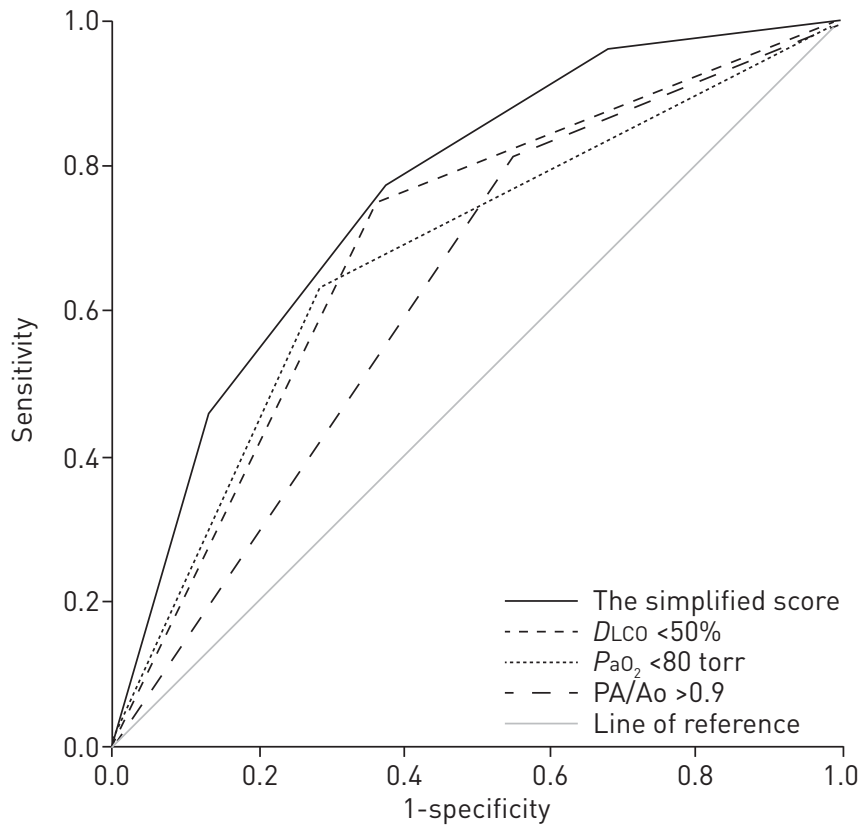

tests, the screening tool presented in this study is simpler, easier to score without special calculations and more easily applied in clinical settings, as each variable is essential for the clinical assessment of IPF.

Regarding the significance of elevated MPAP $(\geqslant 21 \mathrm{mmHg})$, KIMURA et al. [8] have demonstrated a similar prognosis with MPAP between 21 and $24 \mathrm{mmHg}$ and MPAP $>25 \mathrm{mmHg}$ in IPF patients. YAGI et al. [12] have also demonstrated that elevated MPAP is associated with a poorer prognosis in IPF patients. Elevated MPAP has also been indicated to affect survival in interstitial pneumonias other than IPF [24, 25]. Given these findings, detection of elevated MPAP is an important prognostic indicator. Moreover, IPF is known as a heterogeneous disease [1] and elevated MPAP could be used as a specific spectrum to indicate a poorer prognosis. Its early detection would therefore facilitate future research into its clinical significance and that of early interventions.

We have demonstrated that the prevalence of elevated MPAP for scores of zero, one, two and three points is $6.7 \%, 16.0 \%, 29.1 \%$ and $65.4 \%$, respectively. Moreover, the group with a score of three points has a specificity of $95.8 \%$ for elevated MPAP and, as such, it would be reasonable perform a RHC in patients with such a score. The probability of elevated MPAP in a patient with a score of two points is about $30 \%$ and indications for RHC should thus be based on patient preference. We have also shown that there is little value in performing RHC in patients with score of zero because this score has a PPV of only $6.7 \%$ for elevation of MPAP. As such, in our cohort, this combination of noninvasive examinations provides for easy selection of patients for RHC.

The development of PH in IPF is caused mainly by chronic hypoxaemic vasoconstriction and destruction of the vascular bed by progressive fibrosis [4], and, although initially reversible, these mechanisms can eventually cause irreversible vascular remodelling. A reduced DLCO (\% predicted) measurement, which is reflective of fibrosis, destruction of the vascular bed and perfusion inhomogeneity [26], is well recognised to be associated with a higher mortality rate and the presence of PH in IPF [7, 13, 15-17, 21-23]. In advanced IPF, the optimal cut-off value for predicting $\mathrm{PH}$ is reportedly $30 \%$ to $45 \%[15,16,21,27]$. As

\begin{tabular}{|c|c|c|}
\hline Scores $n$ & OR $(95 \% \mathrm{CI})$ & p-value \\
\hline 0 & Reference & \\
\hline 1 & $2.676(1.015-7.057)$ & 0.047 \\
\hline 2 & 5.744 (2.194-15.039) & $<0.001$ \\
\hline 3 & $26.444(8.681-80.556)$ & $<0.001$ \\
\hline
\end{tabular}


TABLE 4 Sensitivity, specificity, positive predictive value (PPV) and negative predictive value (NPV) for elevation of mean pulmonary arterial pressure

\begin{tabular}{lcccc} 
Scores n & Sensitivity $\%$ & Specificity $\%$ & PPV $\%$ & NPV \% \\
\hline $\mathbf{0}$ & 13.2 & 54.2 & 6.7 & 71.6 \\
$\mathbf{1}$ & 24.5 & 68.2 & 16.0 & 78.5 \\
$\mathbf{2}$ & 30.2 & 81.8 & 29.1 & 82.5 \\
$\mathbf{3}$ & 32.1 & 95.8 & 65.4 & 85.1 \\
\hline
\end{tabular}

such, given we were attempting to predict elevated MPAP at initial evaluation, a value of $50 \%$ was selected in consideration of ease-of-use in clinical settings.

A lower $\mathrm{PaO}_{2}$ measurement at rest is also reportedly related to $\mathrm{PH}$ in IPF but the optimal cut-off value is unknown $[8,11,28]$. In the present study, we set the cut-off value as 80 Torr (under the normal limit) based on calculations from the ROC analysis. Moreover, $\mathrm{PaO}_{2}<80$ Torr has been reported as a significant predictor of mortality in IPF patients, giving this value some validity [29].

As for the PA/Ao ratio in IPF, YAGI et al. [12] recently demonstrated that a PA/Ao ratio $\geqslant 0.9$ was a predictor for elevated MPAP, while ALKuKHun et al. [11] demonstrated (in an evaluation for lung transplantation) that a PA/Ao ratio $\geqslant 1.1$ was a predictor for $\mathrm{PH}$. Moreover, the PA/Ao ratio was reportedly demonstrated to be a predictor of higher mortality and it is thus an important indicator. In the present study, we aimed to predict elevated MPAP at initial evaluation and so we adopted 0.9 as a reference value.

Our findings differed from previous reports that have evaluated PH in IPF. Echocardiography remains the most widely used noninvasive diagnostic tool for the assessment of $\mathrm{PH}$ [7]. Shorter distances and greater desaturation in the 6MWT are reportedly also predictive of underlying $\mathrm{PH}$ [16]. However, in the present study, these aspects showed no predictive value of importance. Earlier studies have reported that the accuracy of echocardiography is low $[7,10,14]$ and our results are consistent with these previous findings. Another reason for the differences observed is due to patient selection. Our entire study cohort consisted of patients who had not received any treatment for IPF or PH at initial evaluation, whereas the previous literature examined patients with advanced IPF

The present study has its limitations as, even though the analysis examines internal validation, it was performed in a single centre with patients of a single race. Thus, the results might not be applicable to other general populations and external validation is needed. Moreover, we excluded patients with PAWP $>15 \mathrm{mmHg}$ under RHC and we could therefore assume that the scoring system might not perform well in real-world cohorts including such patients. It should be noted, however, that similar findings were obtained when such patients were added to the original cohort (data not shown). Furthermore, the present study was affected by selection bias, as the subjects were treatment-free patients at the time of initial evaluation, which was likely representative of patients on the milder side of the disease spectrum compared to those evaluated in the previous literature.

The latest guidelines show that currently there is no specific therapy for $\mathrm{PH}$ associated with lung diseases [7] and no established therapy for patients with elevated MPAP. Further study on when and for whom treatment should be started will be needed. Despite these limitations, this study was the largest single series in IPF with all measurements recorded using RHC, even at initial evaluation, and it presented a means of early detection of elevated MPAP without invasive examination.

We conclude that this simple clinical scoring system comprising DLCO (\% predicted), PA/Ao ratio on HRCT scan and $\mathrm{PaO}_{2}$ is able to predict elevated MPAP in patients with IPF. Further multicentre, international studies are necessary.

\section{Acknowledgements}

Author contributions are as follows: T. Furukawa, Y. Kondoh, H. Taniguchi, M. Yagi and T. Matsuda designed the study. K. Kataoka, T. Furukawa and M. Yagi collected the data. T. Furukawa and M. Ando performed the data analysis. All authors contributed to data interpretation. T. Furukawa, Y. Kondoh and H. Taniguchi wrote the original manuscript. All authors contributed to revisions of the manuscript, provided final approval of the version to be published and agreed to be accountable for all aspects of the work.

\section{References}

1 Raghu G, Collard HR, Egan JJ, et al. An official ATS/ERS/JRS/ALAT statement: idiopathic pulmonary fibrosis: evidence-based guidelines for diagnosis and management. Am J Respir Crit Care Med 2011; 183: 788-824.

2 Seeger W, Adir Y, Barbera JA, et al. Pulmonary hypertension in chronic lung diseases. J Am Coll Cardiol 2013; 62: D109-D116. 
3 Smith JS, Gorbett D, Mueller J, et al. Pulmonary hypertension and idiopathic pulmonary fibrosis: a dastardly duo. Am J Med Sci 2013; 346: 221-225.

4 King CS, Nathan SD. Idiopathic pulmonary fibrosis: effects and optimal management of comorbidities. Lancet Respir Med 2017; 5: 72-84.

5 Hayes D Jr, Black SM, Tobias JD, et al. Influence of pulmonary hypertension on patients with idiopathic pulmonary fibrosis awaiting lung transplantation. Ann Thorac Surg 2016; 101: 246-252.

6 Hoeper MM, Bogaard HJ, Condliffe R, et al. Definitions and diagnosis of pulmonary hypertension. J Am Coll Cardiol 2013; 62: D42-D50.

7 Galie N, Humbert M, Vachiery JL, et al. 2015 ESC/ERS Guidelines for the diagnosis and treatment of pulmonary hypertension. Eur Respir J 2015; 46: 903-975.

8 Kimura M, Taniguchi $\mathrm{H}$, Kondoh $\mathrm{Y}$, et al. Pulmonary hypertension as a prognostic indicator at the initial evaluation in idiopathic pulmonary fibrosis. Respiration 2013; 85: 456-463.

9 Teramachi R, Taniguchi $\mathrm{H}$, Kondoh $\mathrm{Y}$, et al. Progression of mean pulmonary arterial pressure in idiopathic pulmonary fibrosis with mild to moderate restriction. Respirology 2017; 22: 986-990.

10 Nathan SD, Shlobin OA, Barnett SD, et al. Right ventricular systolic pressure by echocardiography as a predictor of pulmonary hypertension in idiopathic pulmonary fibrosis. Respir Med 2008; 102: 1305-1310.

11 Alkukhun L, Wang XF, Ahmed MK, et al. Non-invasive screening for pulmonary hypertension in idiopathic pulmonary fibrosis. Respir Med 2016; 117: 65-72.

12 Yagi M, Taniguchi H, Kondoh Y, et al. CT-determined pulmonary artery to aorta ratio as a predictor of elevated pulmonary artery pressure and survival in idiopathic pulmonary fibrosis. Respirology 2017; 22: 1393-1399.

13 Raghu G, Nathan SD, Behr J, et al. Pulmonary hypertension in idiopathic pulmonary fibrosis with mild-to-moderate restriction. Eur Respir J 2015; 46: 1370-1377.

14 Modrykamien AM, Gudavalli R, McCarthy $\mathrm{K}$, et al. Echocardiography, 6-minute walk distance, and distance-saturation product as predictors of pulmonary arterial hypertension in idiopathic pulmonary fibrosis. Respir Care 2010; 55: 584-588.

15 Nathan SD, Shlobin OA, Ahmad S, et al. Pulmonary hypertension and pulmonary function testing in idiopathic pulmonary fibrosis. Chest 2007; 131: 657-663.

16 Lettieri CJ, Nathan SD, Barnett SD, et al. Prevalence and outcomes of pulmonary arterial hypertension in advanced idiopathic pulmonary fibrosis. Chest 2006; 129: 746-752.

17 Zisman DA, Karlamangla AS, Kawut SM, et al. Validation of a method to screen for pulmonary hypertension in advanced idiopathic pulmonary fibrosis. Chest 2008; 133: 640-645.

18 Miller MR, Hankinson J, Brusasco V, et al. Standardisation of spirometry. Eur Respir J 2005; 26: 319-338.

19 Laszlo G. Standardisation of lung function testing: helpful guidance from the ATS/ERS Task Force. Thorax 2006; 61: 744-746.

20 ATS Committee on Proficiency Standards for Clinical Pulmonary Function Laboratories. ATS statement: guidelines for the six-minute walk test. Am J Respir Crit Care Med 2002; 166: 111-117.

21 Trip P, Nossent EJ, de Man FS, et al. Severely reduced diffusion capacity in idiopathic pulmonary arterial hypertension: patient characteristics and treatment responses. Eur Respir J 2013; 42: 1575-1585.

22 Nadrous HF, Pellikka PA, Krowka MJ, et al. Pulmonary hypertension in patients with idiopathic pulmonary fibrosis. Chest 2005; 128: 2393-2399.

23 Zisman DA, Ross DJ, Belperio JA, et al. Prediction of pulmonary hypertension in idiopathic pulmonary fibrosis. Respir Med 2007; 101: 2153-2159.

24 Takahashi $\mathrm{K}$, Taniguchi $\mathrm{H}$, Ando $\mathrm{M}$, et al. Mean pulmonary arterial pressure as a prognostic indicator in connective tissue disease associated with interstitial lung disease: a retrospective cohort study. BMC Pulm Med 2016; 16: 55.

25 Suzuki A, Taniguchi H, Watanabe N, et al. Significance of pulmonary arterial pressure as a prognostic indicator in lung-dominant connective tissue disease. PLoS One 2014; 9: e108339.

26 van der Lee I, Zanen P, Grutters JC, et al. Diffusing capacity for nitric oxide and carbon monoxide in patients with diffuse parenchymal lung disease and pulmonary arterial hypertension. Chest 2006; 129: 378-383.

27 Patel NM, Lederer DJ, Borczuk AC, et al. Pulmonary hypertension in idiopathic pulmonary fibrosis. Chest 2007; 132: 998-1006.

28 Papakosta D, Pitsiou G, Daniil Z, et al. Prevalence of pulmonary hypertension in patients with idiopathic pulmonary fibrosis: correlation with physiological parameters. Lung 2011; 189: 391-399.

29 Homma S, Sugino K, Sakamoto S. Usefulness of a disease severity staging classification system for IPF in Japan: 20 years of experience from empirical evidence to randomized control trial enrollment. Respir Investig 2015; 53: $7-12$. 